\title{
Evidence in oyster of a plasma extracellular superoxide dismutase which binds LPS
}

\author{
Marcelo Gonzalez ${ }^{1}$, Bernard Romestand ${ }^{1}$, Julie Fievet ${ }^{1}$, Arnaud Huvet ${ }^{2}$, Marie-Christine \\ Lebart $^{3}$, Yannick Gueguen ${ }^{1}$, Evelyne Bachère ${ }^{1,{ }^{*}}$
}

\begin{abstract}
${ }^{1}$ UMR 5171, CNRS-UMII-IFREMER, Génome Population Interactions Adaptation, Université de Montpellier II, 2 Place Eugène Bataillon, CC80, 34095 Montpellier Cedex 5 (France)

${ }^{2}$ UMR Physiologie et Ecophysiologie des Mollusques Marins, Centre de Brest, BP 70, 29280 Plouzané, France.

${ }^{3}$ UMR 5539, EPHE, Laboratoire de Motilité Cellulaire, Université Montpellier II, 2 Place Eugène Bataillon, CC 107, 34095 Montpellier Cedex 5 France
\end{abstract}

*: Corresponding author : E. Bachère, email address : evelyne.bachere@ifremer.fr

\begin{abstract}
:
We have characterized in the oyster Crassostrea gigas an extracellular superoxide dismutase (CgEcSOD) which appears to bind lipopolysaccharides (LPS). The protein has been purified from the oyster plasma and identified as a Cu/ZnSOD according to its $\mathrm{N}$-terminal sequencing and biological activity. Cg-EcSOD expression and synthesis are restricted to hemocytes as revealed by in situ hybridization and immunocytochemistry. Cg-EcSOD-expressing hemocytes were seen in blood circulation, in connective tissues, and closely associated to endothelium blood vessels. Cg-EcSOD presents in its amino acid sequence a LPS-binding motif found in the endotoxin receptor CD14 and we show that the protein displays an affinity to Escherichia coli bacteria and with LPS and Lipid A. Additionally, an RGD motif known to be implicated in the association to membrane integrin receptor is present in the amino acid sequence. The purified Cg-EcSOD was shown to bind to oyster hemocytes and to be immunocolocalized with a $\beta$-integrin-like receptor.
\end{abstract}

Keywords: Mollusc bivalve; Invertebrate; Crassostrea gigas; Hemocyte; Oxidative burst; $\beta$-Integrin 


\section{Introduction}

The superoxide dismutases (SODs) are metalloenzymes that represent one important line of defences against reactive oxygen species (ROS) and in particular superoxide anion, resulting from aerobic metabolism. These enzymes catalyse the dismutation of superoxide $\left(\mathrm{O}_{2}{ }^{-}\right)$into molecular oxygen and hydrogen peroxide $\left(\mathrm{H}_{2} \mathrm{O}_{2}\right)$ [1]. The production of ROS can be beneficial as killing mechanism against invading pathogens through the activation of the respiratory burst, a phenomenon present in both vertebrates and invertebrates, such as gastropods [2, 3] and bivalve molluscs [4]. Additionally, it has been shown that ROS, and particularly $\mathrm{H}_{2} \mathrm{O}_{2}$, may serve as second messengers in signal transduction pathway by activating the NF-kappa-B transcription factor [5]. However, elevated concentrations of ROS can be deleterious for the tissues. The SODs are present in both prokaryotic and eukaryotic organisms, invertebrates and vertebrates. In animals, there are two distinct groups of SODs classified depending on the metal content in the active sites, i.e. manganese SOD (MnSOD) restricted to mitochondrial matrix [6]; copper/zinc SODs $(\mathrm{Cu} / \mathrm{Zn}-\mathrm{SOD})$ found primarily in intracellular cytoplasmic compartments or localised to extracellular elements. Cytosolic $\mathrm{Cu} / \mathrm{Zn}$-SODs have a highly conserved structure, widespread in eukaryotes [7]. They are often constitutively expressed and composed of two $16 \mathrm{kDa}$ subunits. In invertebrates, they have been characterized in insects [8] and in molluscs, the gastropod Biomphalaria glabrata [9], and the oyster Crassostrea gigas [10]. In the oyster, the expression of the cytosolic $\mathrm{Cu} / \mathrm{Zn}-$ SOD would be modulated upon hydrocarbon exposure [10]. The extracellular SOD (EcCu/Zn SODs) differs from the cytoplasmic $\mathrm{Cu} / \mathrm{Zn} \mathrm{SOD}$ by the presence of an N-terminal signal cleavage peptide that routes the molecule for secretion [11]. In invertebrates, $\mathrm{EcCu} / \mathrm{Zn}$ SODs have been characterized in several species of parasitic nematodes such as Brugia pahangi [12], Onchocerca volvulus [13] and Caenorhabditis elegans [14]. In crustaceans, the blue crab Callinectes sapidus has an $\mathrm{EcCu} / \mathrm{Zn}$ SOD present in the hemolymph and expressed in hemocytes [15]. Interestingly, in the crayfish Pacifastacus leniusculus, an $\mathrm{EcCu} / \mathrm{Zn}$ SOD has been shown to interact with peroxinectin, a plasma cell-adhesive peroxidase which binds to the surface of the hemocytes. It was proposed that this interaction, SOD-peroxinectin, might mediate hemocyte reactions such as cell adhesion and phagocytosis [16]. In mollusc bivalves, $\mathrm{Cu} / \mathrm{Zn}$ SOD have only been identified at the level of activity and protein in the digestive gland of mussel (Mytilus edulis) [17], whereas in the oyster Crassostrea gigas, $\mathrm{Cu} / \mathrm{Zn}$ SOD 
sequence has been evidenced by suppression subtractive hybridization from the mantle-gonad tissue [18].

Here, we have purified the major protein from the oyster $C$. gigas plasma and identified as being an extracellular $\mathrm{Cu} / \mathrm{Zn}$ SOD $(C g$-EcSOD) similar to the sequence previously identified in mantle-gonad, according to its $\mathrm{N}$-terminal sequencing and biological activity. We show that the extracellular $\mathrm{Cu} / \mathrm{Zn}$ SOD gene expression is restricted to hemocytes, located in the oyster blood vessels and connective tissues. Additionally, the plasma protein appears to bind bacteria, lipopolysaccharides (LPS) and Lipid A, a property which is reinforced by the presence in its amino acid sequence of a LPS-binding motif. Finally, because $C g$-EcSOD sequence contains also an RGD motif, we have investigated the potential binding of this plasma protein to hemocyte through the presence of a $\beta$-integrin-like receptor that we evidence here by confocal microscopy immunolocalisation. This is the first report of an EcSOD both acting as adhesion molecule and involved in LPS-binding. 


\section{Materials and methods}

Animals and hemolymph collection.

Adult oysters, Crassostrea gigas, were purchased from a local oyster farm in PalavasLes Flots (Gulf of Lion, France) and kept in sea water at $15^{\circ} \mathrm{C}$. Hemolymph was collected from the pericardial cavity through the adductor muscle under an equal volume of antiaggregant buffer modified Alsever solution [4] and immediately centrifuged at $1000 \mathrm{~g}$ for $10 \mathrm{~min}\left(4^{\circ} \mathrm{C}\right)$ for obtaining hemocytes.

\section{Protein extraction and $\mathrm{Cg}-\mathrm{EcSOD}$ purification.}

Plasma samples (cell-free hemolymph) collected without antiaggregant solution from 20 oysters were pooled and $50 \mu \mathrm{g}$ of protein was applied to Reverse Phase High Performance Liquid Chromatography (C-18 RP-HPLC column, 2x150 mm, Waters Associates), previously equilibrated with $0.05 \%(\mathrm{v} / \mathrm{v})$ Trifluoroacetic Acid Acidified (TFA) water. A first purification step was performed with a linear gradient of $5-100 \%$ acetonitrile in TFA water, over 30 min at a flow rate of $1 \mathrm{ml} / \mathrm{min}$. Absorbance was monitored at $225 \mathrm{~nm}$. The active fraction was pooled, lyophilized and reconstituted in sterilized water. Then, the second HPLC purification step was performed using the same column and $C g$-EcSOD was eluted with a $5-100 \%$ acetonitrile gradient developed over $50 \mathrm{~min}$.

Protein fractions containing $C g$-EcSOD were identified by SDS-PAGE under reducing condition to evaluate the homogeneity of the preparation. Purified $C g$-EcSOD was incubated without and with $25 \mathrm{mM}$ DTT in $50 \mathrm{mM}$ Tris- $\mathrm{HCl}, 50 \mathrm{mM} \mathrm{NaCl}, \mathrm{pH} 7.5$ buffer for $30 \mathrm{~min}$ at $25^{\circ} \mathrm{C}$. Then, $\mathrm{Cg}$-EcSOD was alkylated with iodoacetamide for $30 \mathrm{~min}$ at room temperature and subjected to SDS/PAGE. Protein concentrations were quantified using micro BCA Protein Assay Reagent Kit (Pierce ${ }^{\mathrm{TM}}$ ) and Bovine Serum Albumin (BSA) was used as a standard. N-terminal sequence analysis of purified $C g$-EcSOD, previously subjected to SDSPAGE and transferred to polyvinylidene difluoride (PVDF) membrane, was performed by automated Edman degradation and detection of the phenylthiohydantoin (PTH) derivates on an automated Procise Applied Biosystems Sequencer.

\section{Antibodies, immunodetection and confocal microscopy}

A Balb/C mouse was immunised by 3 subcutaneous injections of the purified $\mathrm{Cg}$ EcSOD $(20 \mu \mathrm{g})$ diluted in PBS and ascite was collected 2 weeks after intraperitoneal injection with $5 \times 10^{6}$ mice tumor cells in $500 \mu 1$ RPM 1640 (Gibco). Immunoglobulins G (IgG) were purified from ascitic fluid on a Hitrap protein G sepharose column (Pharmacia). Antibodies 
directed against a peptide (sequence KLSDLREYRRFEKEKLKS) chosen in the cytoplasmic domain of the human $\beta 2$ integrin chain were elicited in New Zealand rabbits as described [19]. Anti-peptide antibodies were purified by affinity chromatography on the related peptide as immunoadsorbent [20].

Native $C g$-EcSOD was detected in hemocytes by immunocytochemistry according to the method previously described [21]. Briefly, hemocytes cytocentrifuged on slides were permeabilized with $0.1 \%$ Triton X-100 and successively incubated overnight with anti- $\mathrm{Cg}$ EcSOD polyclonal antibody purified IgG $(4 \mu \mathrm{g} / \mathrm{ml})$. Alkaline phosphatase (PAL)-conjugated goat anti-mouse IgG (Jackson Immuno Research Laboratories, USA) was incubated for $1 \mathrm{~h}$ at room temperature, followed by a $1 \mathrm{~h}$ incubation in the dark in a solution of $100 \mathrm{mM}$ Tris- $\mathrm{HCl}$, $100 \mathrm{mM} \mathrm{NaCl}, 50 \mathrm{mM} \mathrm{MgCl} 2$, $\mathrm{pH} 9.5$ containing $0.19 \mathrm{mg} / \mathrm{ml}$ 5-Bromo-4-chloro-3-indolyl Phosphate (BCIP, Sigma), $0.4 \mathrm{mg} / \mathrm{ml}$ Nitro Blue Tetrazolium (NBT, Sigma) and $0.24 \mathrm{mg} / \mathrm{ml}$ levamisole (Sigma).

For co-localisation of $\beta$-integrin and $C g$-EcSOD, cytocentrifuged hemocytes were incubated for $1 \mathrm{~h}$ at room temperature with anti- $\beta$ integrin cytoplasmic domain $\operatorname{IgG}(4 \mu \mathrm{g} / \mathrm{ml})$ and anti- $C g$-EcSOD IgG, followed by goat anti rabbit FITC and Texas red-conjugated goat anti-mouse IgG (Jackson Immunoresearch) diluted at 1:500 in PBS-Tween 20 (PBS-T) 0.1\% containing 0.005\% Evan's blue (Sigma Diagnostics), respectively. Then, the slides were washed and observed by confocal microscopy Leica TCS 4D. Percentages of hemocytes labelled respectively by anti- $\beta$ integrin $\operatorname{IgG}$ and anti- $C g$-EcSOD $\operatorname{IgG}$ were deducted from confocal observation of 480 hemocytes. For the visualization of colocalized pixels, the plugin of Image J software (NIH) has been used.

Binding of purified $C g$-EcSOD on hemocytes was studied by immunodetection of the molecule on non-permeabilized cytocentrifuged hemocytes. After incubation of the cells with or without purified $C g$-EcSOD $(10 \mu \mathrm{g})$ for $30 \mathrm{~min}$, the hemocytes were washed twice with PBS and incubated for $1 \mathrm{~h}$ with the mouse anti-Cg-EcSOD IgG $(4 \mu \mathrm{g} / \mathrm{ml})$, then with (PAL)conjugated goat anti-mouse $\mathrm{IgG}$ for immunodetection as described above. Percentage of positive cells was determined among 600 randomly chosen hemocytes per conditions. Statistical analyses were carried out using Student's test and a p-value less than 0.05 was considered as significant.

For all these experiments, controls were incubations of specific IgGs preabsorbed by purified protein and the absence of secondary antibodies cross-reactivity was controlled by omitting the primary antibodies. 
SOD activity assay.

SOD activity of the purified plasmatic protein in non-denaturating SDS-PAGE gels was first detected by staining with nitroblue tetrazolium (NBT) according to the method of Beauchamp and Fridovich [22]. Additionnally, the SOD inhibitory activity on hemocyte respiratory burst was studied according to NBT reduction assay as described by Muñoz et al. [23]. Briefly, $100 \mu 1$ of hemolymph containing $5 \times 10^{5}$ hemocytes was distributed per well, and $50 \mu \mathrm{l}$ of zymosan A $\left(1.43 \times 10^{8}\right.$ particles $\left./ \mathrm{ml}\right)$ was used at a 10:1 ratio (zymosan:hemocyte) as elicitor of respiratory burst. In some wells, purified $C g$-EcSOD $(10 \mu \mathrm{g})$ or plasma were added. NBT $(0.3 \%)$ working solutions were immediately distributed to the wells. After $2 \mathrm{~h}$ incubation, the supernatants were removed and the hemocytes were fixed with methanol, washed with methanol $70 \%$ and dried. The formazan deposits were solubilized in $2 \mathrm{M} \mathrm{KOH}$ and DMSO and, after homogenization, the OD at $620 \mathrm{~nm}$ was recorded in a microplate reader. Experiments were performed in triplicates and results are reported as mean \pm standard error (SE) of the mean. Statistical analysis was performed using one-way ANOVA with the Newman-Keuls post test (STATISTICA; StatSoft, Inc). A p-value less than 0.05 was considered as significant.

\section{LPS-binding properties.}

$C g$-EcSOD LPS-binding properties were investigated as described previously by Mannion et al. [24]. Briefly, E. coli K 1/r [25] bacteria were resuspended in $1 \mathrm{ml}$ of $10 \mathrm{mM}$ sodium acetate/acetic acid ( $\mathrm{NaAc} / \mathrm{HAc}$ ) buffer, $\mathrm{pH} 4.0$ and incubated 10 min with $100 \mu \mathrm{g}$ protein with gentle agitation $\left(4^{\circ} \mathrm{C}\right)$. E. coli cells were washed twice by centrifugation with 500 $\mu \mathrm{l}$ of $\mathrm{NaAc} / \mathrm{HAc}$ buffer and bound proteins were subsequently eluted with $30 \mu \mathrm{l}$ of $200 \mathrm{mM}$ $\mathrm{MgCl}_{2}$ in $\mathrm{NaAc} / \mathrm{HAc}$ buffer $\mathrm{pH} 4.0$ and further subjected to SDS-PAGE and HPLC analyses as described above.

Alternatively, LPS-binding properties of purified $C g$-EcSOD were verified by ELISA assay using mouse anti- $C g$-EcSOD polyclonal antibody. E. coli 026:B6 LPS or Lipid A (2.5 $\mu \mathrm{g} /$ well) (Sigma) were coated on a microtiter plate for $2 \mathrm{~h}$ at $60^{\circ} \mathrm{C}$. After $1 \mathrm{~h}$ blocking with PBS buffer containing 5\% BSA, the solid phase was incubated for $60 \mathrm{~min}$ with increasing amounts of purified $C g$-EcSOD (from 0 to $200 \mu \mathrm{g} /$ well). After washing with PBS buffer, the ELISA assay was developed by successive incubation with mouse anti- $C g$-EcSOD polyclonal antibody $(10 \mu \mathrm{g} / \mathrm{ml})$ for $2 \mathrm{~h}$ and with peroxidase-conjugated anti-mouse $\operatorname{IgG}(0.4 \mu \mathrm{g} / \mathrm{ml})$. Controls consisted in the omission of specific antibodies. The colorimetric reaction was initiated by adding $50 \mu \mathrm{l}$ per well of orthophenylene diamine chromogen $(0.4 \mathrm{mg} / \mathrm{ml})$ in 
substrate buffer $\left(0.1 \mathrm{M}\right.$ citric acid, $0.1 \mathrm{M}$ sodium acetate, $\mathrm{pH} 5.4$ in $\left.0.33 \% \mathrm{H}_{2} \mathrm{O}_{2}\right)$. The reaction was stopped after 15 min by adding $25 \mu \mathrm{lof} \mathrm{H}_{2} \mathrm{SO}_{4}$ to the wells. Optical density (OD) was recorded in a microplate reader at $492 \mathrm{~nm}$. All steps were performed at room temperature and two washes with PBS-T were performed between each step. Experiments were performed in triplicates for statistical analyses. The binding parameters, apparent dissociation constant $K \mathrm{~d}$ and the maximum binding $\left(\mathrm{A}_{\max }\right)$ were determined by nonlinearly fitting as $\mathrm{A}=\mathrm{A}_{\max }[\mathrm{L}] /(\mathrm{Kd}+[\mathrm{L}])$, where $\mathrm{A}$ is the absorbance at $492 \mathrm{~nm}$ and $[\mathrm{L}]$ is the ligand concentration by using the CURVE FIT software developed by K. Raner Software (Victoria, Australia) [26].

\section{RT-PCR and molecular cloning.}

Total RNA was extracted from $C$. gigas hemocytes using Trizol reagent according to manufacturer instructions (Invitrogen ${ }^{\mathrm{TM}}$ ) and treated with DNAse Turbo (Ambion). Following heat denaturation $\left(70^{\circ} \mathrm{C}\right.$ for $\left.5 \mathrm{~min}\right)$, reverse transcriptions were performed using $1 \mu \mathrm{g}$ of total RNA prepared with $50 \mathrm{ng} / \mu \mathrm{l}$ oligo- $(\mathrm{dT})_{12-18}$ in a $50 \mu \mathrm{l}$ reaction volume containing $1 \mathrm{mM}$ dNTPs, 1 unit/ $\mu 1$ of RnaseOUT ${ }^{\text {TM }}$ (Invitrogen) and 200 units/ $\mu 1$ M-MLV reverse transcriptase in reverse transcriptase buffer. The cDNAs were amplified using primers EcSODfw 5, AGAGAATCCTGAGCTACAGC 3' and EcSODrev, 5' TGAGCAAAACTCTCTACAAGC 3' designed in the untranslated region of the cDNA sequence (AY551094). The amplification program consisted of a $5 \mathrm{~min}$ at $94^{\circ} \mathrm{C}$, followed by 30 cycles of $94^{\circ} \mathrm{C}$ for $30 \mathrm{~s}, 55^{\circ} \mathrm{C}$ for $30 \mathrm{~s}$, $72^{\circ} \mathrm{C}$ for $1 \mathrm{~min}$ and a final elongation step of $72^{\circ} \mathrm{C}$ for $10 \mathrm{~min}$. Amplified products were analyzed on $1 \%$ agarose gels, cloned into pCR 2.1 TOPO TA cloning vector (Invitrogen) and sequenced from both directions with $\mathrm{T} 7$ and $\mathrm{T} 3$ primers.

\section{In situ hybridization.}

Tissues and hemocytes from $C$. gigas oyster were prepared for histology and in situ hybridization analyses as described by Muňoz et al (2002) [21]. The plasmid containing $C g$ EcSOD cDNA (Genbank accession number DQ010420) was used as template for the preparation of the probes. Digoxigenin (DIG)-UTP labelled antisense and sense riboprobes were generated from linearized cDNA plasmids by in vitro transcription using RNA labelling kits, T3 RNA polymerase (Roche). DIG-labelled riboprobes were hybridized both to oyster tissues and to cytocentrifuged hemocyte preparations as described previously [21] for determination of percentage of $C g$-EcSOD-expressing circulating hemocytes. Control consisted in replacing antisense riboprobe with sense riboprobe. 
Sequence analysis.

Homology searches were performed with the BLAST software on the NCBI home page (htp://wwwncbigov/Blast) Deduced amino acid sequences were aligned by ClustalX (http://www.ch.embnet.org/software/ClustalW.html).

\section{Results and discussion}

\section{Isolation of a plasma extracellular $\mathrm{Cu} / \mathrm{ZnSOD}$}

As observed on SDS-PAGE electrophoresis analysis, a protein appears to greatly predominate in the plasma of the oyster Crassostrea gigas (Fig. 1). In an attempt to characterize this major protein, we have performed its purification by reverse phase HPLC from oyster plasma. In SDS-PAGE under non-reducing conditions, the purified protein appears as a single band with an apparent molecular mass of $20 \mathrm{kDa}$. However, following reduction and alkylation treatment, the linearized protein migrates as around a $30 \mathrm{kDa}$ band, suggesting that the $C g$-EcSOD is a monomer with intramolecular disulfide bonds (Fig. 1).

The purified protein was further electrotransferred on PVDF membrane for N-terminal sequencing by Edman degradation and a first 10 amino acid sequence was obtained TARNEANVNI. Searching protein sequence databases, we showed that this plasma protein unequivocally matches with deduced amino acid sequence of SOD (AY551094) previously identified and cloned from mantle-gonad cDNA library [18] (Fig. 2). Oyster SOD consists of 174 amino acids and its sequence appears to be significantly similar to the proteins from the extracellular SOD family while it shows lower identity with the oyster cytosolic SOD (14 \% identity) [10]. The oyster SOD we have isolated shares $20 \%$ amino acid sequence identity with the extracellular human and nematode SODs (Fig. 2) and it was consequently named $\mathrm{Cg}$ EcSOD. $C g$-EcSOD is also very similar (94\% identity) to previously described oyster sequences named cavortin isolated from $C$. gigas oyster from a New Zealand farm (AY256853) but which would be an SOD.

Concordant with sequence similarity to SODs, we demonstrated a SOD activity for the protein purified from the oyster plasma. SOD activity was first detected in non-denaturing gel by NBT reduction assay according to the method of Beauchamp and Fridovich [22]. Activity appeared located on a unique band both in the plasma and for the purified protein (data not shown). SODs are known to catalyse the dismutation of superoxide anion into molecular oxygen and hydrogen peroxide [1]. This antioxidant activity has also been evidenced for the purified $C g$-EcSOD by an inhibitory effect on the ROS production from oyster hemocytes. 
The capacity of oyster hemocytes to generate respiratory burst upon phagocytic stimulation is well documented and various methods have been developed to study this phenomenon [4, 27]. Here, we have used a colorimetric assay based on the detection of formazan deposit resulting from the reduction of NBT by ROS, and previously described for shrimp hemocytes [23]. In our experiments, addition of purified $C g$-EcSOD to oyster hemocytes phagocytosing zymosan particles resulted in an inhibition of ROS production which was measured as the optical density (OD) of solubilized formazan deposit (Fig. 3). While, the phagocytosis of zymosan by C. gigas hemocytes induced the production of superoxide anion corresponding to an OD of 0.25 compared to unstimulated hemocytes (base activity of $0.17 \mathrm{OD}$ ), hemocytes incubated with $C g$-EcSOD displayed a significant reduced reaction with an OD of $0.20(\mathrm{p}<0.05)$ which revealed a dismutation of the superoxide anion. Similar effects have been shown in the assay following the addition of oyster plasma $(0.18 \mathrm{OD} ; \mathrm{p}<0.05)$ which contains huge amount of $C g$-EcSOD (Fig. 3). In bivalve, as other invertebrates, phagocytosis activity is known as an important component of defence reactions, but which may contribute to inflammatory reactions and tissue damage due to the massive release of ROS into extracellular fluids. The oyster has an open circulatory system and the hemolymph is in contact direct and bath connective tissues. It can be assumed that $C g$-EcSOD which is the major plasma protein could play a protective role against inflammatory reaction as established for human phagocytic cells [28]. In other respect, SOD contributes to the production of $\mathrm{H}_{2} \mathrm{O}_{2}$ which in turn is dismutated by catalase. $\mathrm{H}_{2} \mathrm{O}_{2}$ is thought to be an important second messenger [29] and regulator of proliferation of mammalian cells. Low concentrations of $\mathrm{H}_{2} \mathrm{O}_{2}$ stimulate proliferation or enhanced survival of a wide variety of cell types [30,31]. Nothing is known about similar role of $\mathrm{H}_{2} \mathrm{O}_{2}$ and SOD in the activation of hemocyte proliferation which would be a major element of the immune response in invertebrates such as the oyster [32].

\section{Gene expression and production of $\mathrm{Cg}$-EcSOD is restricted to hemocytes.}

We have produced a specific polyclonal antibody for the immunodetection and localisation in oyster tissues of $C g$-EcSOD, previously identified from the gonad and mantle of the oyster [18]. Surprisingly, an immunoreactivity was observed by western blot mainly with circulating hemocytes and in a lesser extent on the tissues cited above (data not shown), questioning about the tissue origin of the plasma $C g$-EcSOD. Consequently, we decided to look at the potential expression of $C g$-EcSOD mRNA in hemocytes.

In a first time, a PCR approach was used to identify and clone the $C g$-EcSOD from hemocytes, considering primers designed from the sequence (AY551094) isolated from the 
mantle-gonad. A single band around $600 \mathrm{pb}$ was obtained, cloned and sequenced. The sequence encompassed a 576-bp open reading coding for a 193-amino-acid protein, which was shown to be homologous (96\%) to the mantle-gonad SOD sequence. The complete $C g$ EcSOD (DQ010420) cDNA encodes a 174 residue protein with the same $\mathrm{N}$-terminal sequence than the purified plasma protein and with an estimated molecular mass of $19.465 \mathrm{Da}$ and a $\mathrm{pI}$ of 4.84. This estimated mass corresponds to the apparent mass of $20 \mathrm{kDa}$ seen in SDS-PAGE under non-reducing condition. As shown for the EcSOD identified in mantle-gonad, the sequence contains an 18-residue signal peptide followed by a mature protein domain as predicted by the Signal P program (http:/www.cbs.dtu.dk/services/SignalP). Interestingly, $C g$-EcSOD possesses in its sequence an RGD integrin-binding motif and has a LPS-binding motif as observed in mammalian CD14 protein [33] (Fig. 2).

$C g$-EcSOD gene expression was studied by in situ hybridization for tissue localisation. Tissue sections of oysters were probed with DIG labelled antisense riboprobes and detected with alkaline phosphatase-conjugated anti-DIG antibodies, whereas sens riboprobes were used as control of specificity (Fig. 4A'). $C g$-EcSOD expression was shown to be unequivocally restricted to hemocytes circulating and infiltrating connective tissues (Fig. 4A). Expressing hemocytes were also observed closely associated to the endothelium of blood vessel in the mantle (Fig. 4B). With respect to in situ hybridization analyses on circulating hemocytes, $C g$-EcSOD transcripts were detected in a few populations of hemocytes with only $7.0 \%$ of positive cells (out of 650 hemocytes observed) (data not shown). These results reveal that the $C g$-EcSOD cDNA sequence previously isolated by genomic approach from the gonad-mantle of C.gigas [18] originated from hemocytes infiltrating this tissue. Additionnally, immunolocalisation of $C g$-EcSOD with specific antibody revealed that around $12.0 \%$ of the total circulating hemocytes (out of 730 observed) were positive when the cell preparations have been membrane-permeabilised before incubation with the specific antibody (data not shown). All together, these results suggested that plasma $C g$-EcSOD could also bind at the hemocyte membrane surface.

\section{Plasma $C g$-EcSOD binds to hemocyte membrane}

To further investigate putative binding of plasma $C g$-EcSOD on the hemocyte surface, purified protein has been incubated with non-permeabilised hemocytes previously to its immunodetection. Significant increase in the number of positive hemocytes $(32.9 \%, \mathrm{p}<0.05)$ (Fig 5B and 5C) was obtained compared to conditions where hemocytes are not incubated with purified $C g$-EcSOD (11.6\% of positive cells)(Fig 5A and $5 \mathrm{C}$ ). In the crayfish, 
Pacifastacus leniusculus, an EcSOD was also shown to be localised at the hemocyte surface and it was additionally recognised as a peroxidase-binding protein [16]. The authors proposed that the EcSOD could be maintained at the cell surface through ionic interactions. In oyster, as previously mentioned, $\mathrm{Cg}$-EcSOD does not have transmembrane region but it presents in its sequence a RGD motif (Fig. 2) which is known to be recognised by integrins that function as cell-cell or cell-substrate adhesion and in signal transduction [34]. These proteins are ubiquitous in vertebrates and invertebrates. They are involved in several immune functions, including cell adhesion, motility and cell proliferation $[35,36]$. In invertebrates, hemocyte $\beta$ integrins have been shown to be involved in phagocytosis processes [37]. The presence of a RGD motif in the $C g$-EcSOD sequence prompted us to investigate the presence of $\beta$ integrin in oyster hemocytes and its potential co-localisation with the plasma $C g$-EcSOD. This has been approached using a specific antibody directed against a peptidic sequence chosen in the cytoplasmic domain of $\beta$ integrin which is conserved among vertebrates [38]. In our experiments, merged confocal microscope images revealed that $\beta$ integrin and $C g$-EcSOD were detected in different circulating hemocytes with $1.8 \%$ of cells immunoreactive to $\beta$ integrin (Fig. 5D) and $7.1 \%$ for $C g$-EcSOD (Fig. 5E), respectively. However, for $2.9 \%$ of the cell populations, $\beta$ integrin and $C g$-EcSOD were shown to be closely associated within same hemocytes as shown by merged confocal images (Fig. 5F). To facilitate the visualization of the merged image, the colocalised pixels appear in white and the pixel colocalisation was estimated with a threshold of 5\% (Fig. 5G). Additionally, to reinforce this result of an interaction of $C g$-EcSOD on hemocyte via a $\beta$ integrin receptor, we evidenced in our $C$. gigas hemocyte Expression Sequence Tag (EST) database (httpp://www.ifremer.fr/GigasBase), two clones (GenBank BQ42637; BQ426447) which present sequence homologies with $\beta$ integrins from Mus musculus and the jellyfish Podocoryne carnea. All together, these findings argue in favour of a binding of the plasma $C g$-EcSOD on some hemocyte population via an $\beta$ integrinRGD interaction for further activating cellular reactions or immune processes.

\section{The oyster $C g$-EcSOD binds to bacteria, LPS and Lipid A.}

As previously mentioned, $C g$-EcSOD displays in its N-terminal sequence a LPSbinding motif, DDED (Fig. 2), which is known to be involved in the fixation of LPS on the endotoxin receptor CD14 $[33,39]$. To approach the role of this motif in the oyster plasma protein, we have investigated the potential property of both the plasma and the purified $\mathrm{Cg}$ EcSOD to bind LPS. For that, E. coli bacteria were incubated with C. gigas plasma and, after 
several washes, two bounded proteins were recovered by magnesium chloride elution as revealed by RP-HPLC and SDS-PAGE analysis. Beside a minor undetermined plasma protein of a molecular mass of around $60 \mathrm{kDa}, C g$-EcSOD was evidenced to be eluted (Data not shown). This property of purified $C g$-EcSOD was further verified against soluble LPS and Lipid A. Increasing amounts of purified $C g$-EcSOD have been added to immobilized LPS or Lipid A on microplate and, after several washes, bound protein has been detected by ELISA using the specific anti- $C g$-EcSOD antibody. From these assays made in triplicates, we evidenced an increase in absorbance according to the concentration of $C g$-EcSOD that revealed the binding of the protein for both LPS and Lipid A. In wells without LPS or Lipid A, no binding of $C g$-EcSOD has been detected. The apparent constant of dissociation of $\mathrm{Cg}$ EcSOD for LPS $\left(\mathrm{K}_{\mathrm{d}}=1.50 \times 10^{-5}( \pm 0.25) \mathrm{M}\right)$ was almost the same as that obtained for Lipid A $\left(\mathrm{K}_{\mathrm{d}}=1.25 \times 10^{-5}( \pm 0.23) \mathrm{M}\right)$ (Fig 6). Compared to well known LPS-binding proteins such as the Limulus endotoxin binding protein-protease inhibitor, the affinity of the purified $\mathrm{Cg}$ EcSOD for LPS appears to be 1000-fold weaker [40]. However, considering its great abundance in the oyster hemolymph, this property appears to be relevant with a potential role of this circulating protein in LPS or bacteria-binding.

Conclusion. Numerous proteins are known with LPS binding properties [41]. To our knowledge, this is the first evidence of a SOD which appear to display LPS-binding property. Further work must be addressed for characterizing this property and how $C g$-EcSOD may interact with Lipid A, LPS or the bacteria. It is known that recognition of microbial products leads to cell activation and production of a large array of mediators necessary for the establishment of inflammatory processes. LPS recognition mechanisms can be provided by several actors which can interplay such as plasma LBP-binding protein (LBP), membranebound or soluble forms of CD14 and integrins [42]. In vertebrates, LBP would accelerate the binding of LPS to CD14 [43, 44]. In contrast, $\beta 2$ integrin has been shown to only participate in the recognition of particulate form of LPS [42]. This has also been evidenced for insect hemocytes in which $E$. coli is internalized through $\beta_{3}$ integrin-dependent process whereas cell-free LPS is internalized through a receptor-mediated endocytosis [37]. As we observed in our experiments the formation of aggregation with the purified $C g$-EcSOD, we can assume that aggregated $C g$-EcSOD-LPS particles or $C g$-EcSOD bound bacteria may interact with $\beta$ integrin for further activation and internalisation within the oyster hemocytes. For the oyster C. gigas which is permanently in contact with a rich microflora in its environment, it is likely 
that the major plasmatic protein may display different functions related to the elimination of microorganisms including antioxidant effect and LPS-binding property. $C g$-EcSOD may have an opsonic activity by promoting recognition and elimination of bacteria through the binding to hemocyte integrins. However, through the SOD activity, the molecule may participate in the regulation of inflammatory response induced by sepsis by detoxifying internal oyster tissues or by improving the ability of the hemocytes to resist oxidant injury.

\section{Acknowledgements}

The authors are grateful to A. Sahuquet from the UMR 5539 (University of Montpellier 2) for technical assistance and advices for confocal microscopy. Work in the author laboratory is supported by the Ifremer, the CNRS and the University of Montpellier II. This study was part of a collaborative project supported by the European Commission, DG XII, in the program International Co-operation with Developing Countries, INCO-DC, Contract $\mathrm{n}^{\circ}$ ICA4-CT2001-10023 (IMMUNAQUA) and of the MOREST national project funded by Ifremer and by the "Région Basse-Normandie, Bretagne, Pays de la Loire and Poitou-Charentes, and the "Conseil Général du Calvados".

\section{REFERENCES}

[1] I. Fridovich, Superoxide radical and superoxide dismutases, Annu Rev Biochem 64 (1995) 97-112.

[2] R. Dikkeboom, W.P.W. van der Knaap, and E.A. Meuleman, Generation of toxic oxygen metabolites by hemocytes of the snail Lymnaea stagnalis: A potential killing mechanism, Dev. Comp. Immunol. 10 (1986) 633.

[3] C.J. Bayne, U.K. Hahn, and R.C. Bender, Mechanisms of molluscan host resistance and of parasite strategies for survival, Parasitology 123 Suppl (2001) S159-167.

[4] E. Bachère, D. Hervio, and E. Mialhe, Luminol-dependent chemiluminescence by hemocytes of two marine bivalves, Ostrea edulis and Crassostrea gigas, Dis. Aquat. Org. 11 (1991) 173-180. 
[5] R. Schreck, P. Rieber, and P.A. Baeuerle, Reactive oxygen intermediates as apparently widely used messengers in the activation of the NF-kappa B transcription factor and HIV1, Embo J 10 (1991) 2247-2258.

[6] A. Okado-Matsumoto, and I. Fridovich, Subcellular distribution of superoxide dismutases (SOD) in rat liver: Cu,Zn-SOD in mitochondria, J Biol Chem 276 (2001) 38388-38393.

[7] D. Bordo, K. Djinovic, and M. Bolognesi, Conserved patterns in the $\mathrm{Cu}, \mathrm{Zn}$ superoxide dismutase family, J Mol Biol 238 (1994) 366-386.

[8] J.D. Parker, K.M. Parker, and L. Keller, Molecular phylogenetic evidence for an extracellular $\mathrm{Cu} \mathrm{Zn}$ superoxide dismutase gene in insects, Insect Mol Biol 13 (2004) 587594.

[9] C.P. Goodall, R.C. Bender, E.J. Broderick, and C.J. Bayne, Constitutive differences in $\mathrm{Cu} / \mathrm{Zn}$ superoxide dismutase mRNA levels and activity in hemocytes of Biomphalaria glabrata (Mollusca) that are either susceptible or resistant to Schistosoma mansoni (Trematoda), Mol Biochem Parasitol 137 (2004) 321-328.

[10] I. Boutet, A. Tanguy, and D. Moraga, Response of the Pacific oyster Crassostrea gigas to hydrocarbon contamination under experimental conditions, Gene 329 (2004) 147-157.

[11] K. Hjalmarsson, S.L. Marklund, A. Engstrom, and T. Edlund, Isolation and sequence of complementary DNA encoding human extracellular superoxide dismutase, Proc Natl Acad Sci USA 84 (1987) 6340-6344.

[12] L. Tang, X. Ou, K. Henkle-Duhrsen, and M.E. Selkirk, Extracellular and cytoplasmic $\mathrm{CuZn}$ superoxide dismutases from Brugia lymphatic filarial nematode parasites, Infect Immun 62 (1994) 961-967.

[13] E.R. James, D.C. McLean, and F. Perler, Molecular cloning of an Onchocerca volvulus extracellular Cu-Zn superoxide dismutase, Infect Immun 62 (1994) 713-716.

[14] M. Fujii, N. Ishii, A. Joguchi, K. Yasuda, and D. Ayusawa, A novel superoxide dismutase gene encoding membrane-bound and extracellular isoforms by alternative splicing in Caenorhabditis elegans, DNA Res 5 (1998) 25-30.

[15] M. Brouwer, T. Hoexum Brouwer, W. Grater, and N. Brown-Peterson, Replacement of a cytosolic copper/zinc superoxide dismutase by a novel cytosolic manganese superoxide dismutase in crustaceans that use copper (haemocyanin) for oxygen transport, Biochem $\mathbf{J}$ 374 (2003) 219-228.

[16] M.W. Johansson, T. Holmblad, P.O. Thornqvist, M. Cammarata, N. Parrinello, and K. Söderhäll, A cell-surface superoxide dismutase is a binding protein for peroxinectin, a cell-adhesive peroxidase in crayfish., J Cell Sci 112 (1999) 917-925. 
[17] H. Manduzio, T. Monsinjon, B. Rocher, F. Leboulenger, and C. Galap, Characterization of an inducible isoform of the $\mathrm{Cu} / \mathrm{Zn}$ superoxide dismutase in the blue mussel Mytilus edulis, Aquat Toxicol 64 (2003) 73-83.

[18] A. Huvet, A. Herpin, L. Degremont, Y. Labreuche, J.F. Samain, and C. Cunningham, The identification of genes from the oyster Crassostrea gigas that are differentially expressed in progeny exhibiting opposed susceptibility to summer mortality, Gene 343 (2004) 211-220.

[19] Y. Benyamin, C. Roustan, and M. Boyer, Anti-actin antibodies. Chemical modification allows the selective production of antibodies to the N-terminal region, J Immunol Methods 86 (1986) 21-29.

[20] C. Mejean, M.C. Lebart, M. Boyer, C. Roustan, and Y. Benyamin, Localization and identification of actin structures involved in the filamin-actin interaction, Eur J Biochem 209 (1992) 555-562.

[21] M. Munoz, F. Vandenbulcke, D. Saulnier, and E. Bachère, Expression and distribution of penaeidin antimicrobial peptides are regulated by haemocyte reactions in microbial challenged shrimps, Eur. J. Biochem. 269 (2002) 2678-2689.

[22] C. Beauchamp, and I. Fridovich, Superoxide dismutase: improved assays and an assay applicable to acrylamide gels, Anal Biochem 44 (1971) 276-287.

[23] M. Munoz, R. Cedeno, J. Rodriguez, W.P.W. van der Knaap, E. Mialhe, and E. Bachère, Measurement of reactive oxygen intermediate production in haemocytes of the penaeid shrimp, Penaeus vannamei (Decapoda), Aquaculture 191 (2000) 89-107.

[24] B.A. Mannion, E.S. Kalatzis, J. Weiss, and P. Elsbach, Preferential binding of the neutrophil cytoplasmic granule-derived bactericidal/permeability increasing protein to target bacteria. Implications and use as a means of purification, J Immunol 142 (1989) 2807-2812.

[25] O. Levy, C.E. Ooi, P. Elsbach, M.E. Doerfler, R.I. Lehrer, and J. Weiss, Antibacterial proteins of granulocytes differ in interaction with endotoxin. Comparison of bactericidal/permeability-increasing protein, p15s, and defensins, J Immunol 154 (1995) 5403-5410.

[26] M.C. Lebart, F. Hubert, C. Boiteau, S. Venteo, C. Roustan, and Y. Benyamin, Biochemical characterization of the L-plastin-actin interaction shows a resemblance with that of alpha-actinin and allows a distinction to be made between the two actin-binding domains of the molecule, Biochemistry 43 (2004) 2428-2437. 
[27] C. Lambert, P. Soudant, G. Choquet, and C. Paillard, Measurement of Crassostrea gigas hemocyte oxidative metabolism by flow cytometry and the inhibiting capacity of pathogenic vibrios, Fish Shellfish Immunol 15 (2003) 225-240.

[28] M.L. Salin, and J.M. McCord, Free radicals and inflammation. Protection of phagocytosine leukocytes by superoxide dismutase, J Clin Invest 56 (1975) 1319-1323.

[29] M. Reth, Hydrogen peroxide as second messenger in lymphocyte activation, Nat Immunol 3 (2002) 1129-1134.

[30] J.R. Stone, and T. Collins, The role of hydrogen peroxide in endothelial proliferative responses, Endothelium 9 (2002) 231-238.

[31] M. Hachiya, and M. Akashi, Catalase regulates cell growth in HL60 human promyelocytic cells: evidence for growth regulation by $\mathrm{H}(2) \mathrm{O}(2)$, Radiat Res 163 (2005) 271-282.

[32] E. Bachère, Y. Gueguen, M. Gonzalez, J. de Lorgeril, J. Garnier, and B. Romestand, Insights into the antimicrobial defense of marine invertebrates: the penaeid shrimps and the oyster Crassostrea gigas, Immunol. Rev. 198 (2004) 149-168.

[33] T.S. Juan, E. Hailman, M.J. Kelley, S.D. Wright, and H.S. Lichenstein, Identification of a domain in soluble CD14 essential for lipopolysaccharide (LPS) signaling but not LPS binding, J Biol Chem 270 (1995) 17237-17242.

[34] A. Sonnenberg, Integrins and their ligands., Curr Top Microbiol Immunol (1993) 7-35.

[35] M.A. Schwartz, and R.K. Assoian, Integrins and cell proliferation: regulation of cyclindependent kinases via cytoplasmic signaling pathways, J Cell Sci 114 (2001) 2553-2560.

[36] R.L. Juliano, P. Reddig, S. Alahari, M. Edin, A. Howe, and A. Aplin, Integrin regulation of cell signalling and motility, Biochem Soc Trans 32 (2004) 443-446.

[37] L.C. Foukas, H.L. Katsoulas, N. Paraskevopoulou, A. Metheniti, M. Lambropoulou, and V.J. Marmaras, Phagocytosis of Escherichia coli by insect hemocytes requires both activation of the Ras/mitogen-activated protein kinase signal transduction pathway for attachment and beta3 integrin for internalization, J Biol Chem 273 (1998) 14813-14818.

[38] L. Zecchinon, T. Fett, E. Baise, and D. Desmecht, Molecular cloning and characterisation of the CD18 partner in ovine (Ovis aries) beta2-integrins, Gene 334 (2004) 47-52.

[39] R.A. Shapiro, M.D. Cunningham, K. Ratcliffe, C. Seachord, J. Blake, J. Bajorath, A. Aruffo, and R.P. Darveau, Identification of CD14 residues involved in specific lipopolysaccharide recognition, Infect Immun 65 (1997) 293-297. 
[40] N.S. Tan, M.L. Ng, Y.H. Yau, P.K. Chong, B. Ho, and J.L. Ding, Definition of endotoxin binding sites in horseshoe crab factor $\mathrm{C}$ recombinant sushi proteins and neutralization of endotoxin by sushi peptides, Faseb J 14 (2000) 1801-1813.

[41] R. Chaby, Lipopolysaccharide-binding molecules: transporters, blockers and sensors, Cell Mol Life Sci 61 (2004) 1697-1713.

[42] T.H. Flo, L. Ryan, L. Kilaas, G. Skjak-Braek, R.R. Ingalls, A. Sundan, D.T. Golenbock, and T. Espevik, Involvement of CD14 and beta2-integrins in activating cells with soluble and particulate lipopolysaccharides and mannuronic acid polymers, Infect Immun 68 (2000) 6770-6776.

[43] R.I. Tapping, and P.S. Tobias, Cellular binding of soluble CD14 requires lipopolysaccharide (LPS) and LPS-binding protein, J Biol Chem 272 (1997) 2315723164.

[44] E. Hailman, H.S. Lichenstein, M.M. Wurfel, D.S. Miller, D.A. Johnson, M. Kelley, L.A. Busse, M.M. Zukowski, and S.D. Wright, Lipopolysaccharide (LPS)-binding protein accelerates the binding of LPS to CD14, J Exp Med 179 (1994) 269-277. 


\section{Legend Figures}

Fig. 1. Cg-EcSOD purification from Crassostrea gigas plasma. Reverse-phase HPLC profile of $C$. gigas plasma shows one major peak (arrow). Black rectangle means hand fraction collected and analysed by SDS-PAGE and Coomassie blue staining. Lane 1: SDSPAGE profile of crude oyster plasma revealing major protein at around $30 \mathrm{kDa}$ under reducing conditions; lane 2: eluted protein after RP-HPLC purification under reducing conditions appears as a unique band at $30 \mathrm{kDa}$; lane 3: under non-reducing conditions (-DTT) the eluted protein appears as a band corresponding to $20 \mathrm{kDa}$. Molecular mass markers are included on the right.

Fig. 2. Alignment of the oyster superoxide dismutase (Cg-EcSOD) with extracellular superoxide dismutases from some other animals. The accession numbers for these

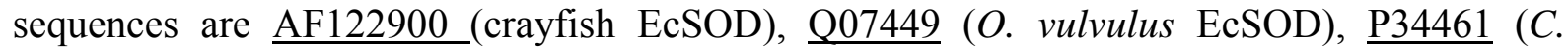

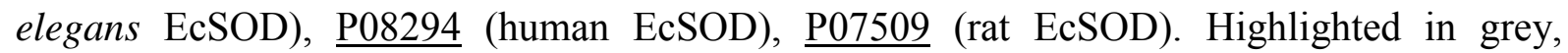
conserved residues; * conserved amino acid residues responsible for $\mathrm{Cu}$ binding; + conserved amino acid residues responsible for $\mathrm{Zn}$ binding. Putative RGD or KGD integrin binding motifs are highlighted in dark. The LPS-binding motif is enclosed in a box. The N-terminal sequence obtained from plasmatic purified $C g$-EcSOD is underlined.

Fig. 3. Inhibitory effect of purified $C g$-EcSOD and plasma on oyster hemocyte respiratory burst. $\mathrm{Cg}$-EcSOD activity has been evidenced by its capacity to inhibit the oyster hemocyte production of ROS using a colorimetric NBT reduction assay. Absorbances are Optical Density of solubilized formazan deposits resulting from NBT reduction. Lane 1: base activity of unstimulated hemocytes incubated with buffer; lane 2: ROS production of hemocytes stimulated with zymosan at a ratio of 10 to 1 hemocyte $(10 / 1)$; lane 3 : unstimulated hemocytes incubated only with purified $C g$-EcSOD $(10 \mu \mathrm{g})$; lane 4 . ROS production of hemocytes stimulated with zymosan (10/1) in presence of purified $C g$-EcSOD $(10 \mu \mathrm{g})$; lane 5: unstimulated hemocytes incubated with oyster crude plasma; lane 6: ROS production of hemocytes stimulated with zymosan (10/1) in presence of oyster crude plasma. The values represent the mean and SE for three independent experiments. Columns with different letters are significantly different $(\mathrm{P}<0.05)$. 
Fig. 4. Detection of $\boldsymbol{C g}$-EcSOD mRNA in oyster tissues by in situ hybridization. (A) In the mantle, hybridization positive signals were located to hemocytes associated with hemolymphatic sinus and infiltrating connective tissues (arrows). (A') Negative control consisted of hybridization with $C g$-EcSOD sense riboprobe. (B) In the hemolymphatic sinus, labelled hemocytes (arrows) are seen associated with the wall blood vessel while free hemocytes observed in the lumen are not labelled. $\mathrm{Ct}$, means connective tissue, Ep: epithelium and S: sinus.

Fig 5. Confocal microscopic images of $\boldsymbol{C g}$-EcSOD (red) and $\beta$ integrin (green) double immune labelling in oyster circulating hemocytes. Immunolocalisation of $C g$-EcSOD on hemocyte cell surface has been determined by incubating non-permeabilized cells without (A) and with purified $C g$-EcSOD (10 $\mu$ g protein) (B); percentage of $C g$-EcSOD-immunoreactive hemocytes has been determined from 600 hemocytes. *statistical difference with respect to incubation with purifed $C g$-EcSOD protein, $\mathrm{P}<0.05(\mathrm{C})$. The values represent the mean and SE for three independent experiments. Merged confocal microscope images suggest that $\beta-$ integrin (green) and $\mathrm{Cg}$-EcSOD (red) are present in different hemocytes (D and E, respectively), but also co-localised inside same hemocytes $(\mathrm{F})$. The colocalized pixels shown in white were determined by Image J software $(G)$.

Fig 6. Binding properties of $\boldsymbol{C g}$-EcSOD to LPS and Lipid A. Increasing concentrations of purifed $C g$-EcSOD (0 to $200 \mu \mathrm{g} / \mathrm{ml}$ ) have been incubated with lipopolysaccharides (LPS) and Lipid A (Lip A) immobilized on microplate and bound protein has been detected by ELISA using specific antibody. The values represent the mean and SE for three independent experiments. 


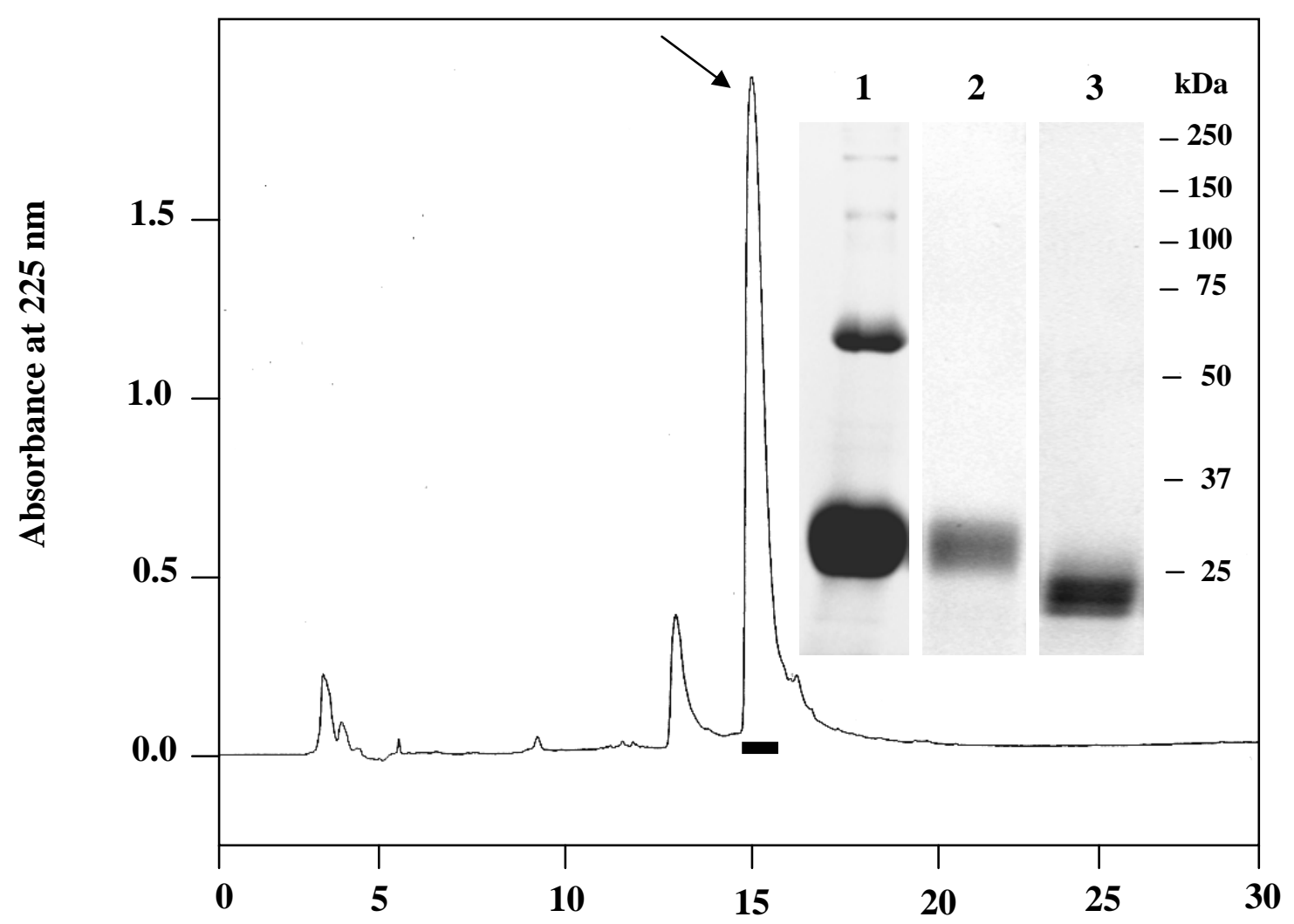

Time (min)

Figure 1 


\section{Figure 2}

Crayfish EcSOD

O. volvulus ECSOD

C. elegans ECSOD

Human ECSOD

Rat ECSOD

Cg-EcSOD

Crayfish EcSOD

O. volvulus ECSOD

C. elegans ECSOD

Human ECSOD

Rat ECSOD

Cg-EcSOD

Crayfish EcSOD

O. volvulus ECSOD

C. elegans ECSOD

Human ECSOD

Rat ECSOD

Cg-EcSOD

Crayfish EcSOD

O. volvulus ECSOD

C. elegans ECSOD

Human ECSOD

Rat ECSOD

Cg-EcSOD
-AVVDLVPGSDQIS

VCVEATHVYGRRSHSNGMHGNGARRAVAVIRGDAGVS

-ASEVIRARAYIFKAEAGKIP--TELI

WTGEDSAEPNSDSAEWIRDMYAKVTEIWQEVMQRRDDDGTLHAACQVQPSATLDAAQPRVI WTMSDTGESGVDLADRLDLVEKIGDTHSKDLEIVMELGKQREADAREMHAVCRVQPSAMLPPDQPQIT TARNEANVNIYLHL DDED,\$NYENSMHYAQCEMEPNHEMPGNLHHRV

\section{LPS-binding domain}

GRLEIYRSYNG--LTIVGTVSGLTPG----KHGFH------VHQKGDLGDGCKAAG--GHFNPFNKNHG GIIYFQQGSGGSITTISGSVSGLTPG----_LHGFH-----VHQYGDQTNGCTSAG--DHYNPFGKTHG GTIDFDQ--SGSFLKLNGSVSGLAAG----KHGFH-----IHEKGDTGNGCLSAG--GHYNPHKLSHG GVVLFRQLAPRAKLDAFFALEGFPTEPNSSSRAIH-----VHQFGDLSQGCESTG--PHYNPLAVPHP GLVLFRQLGPSSRLEASFNLEGFPAEQNTSNHAIH-----VHEFGDLSQGCESTG--PHYNPLGVPHP HGSIEMHQRGDGPLEMEFHLTGFNVSEDFAD---HNYGLQIHEYGDLEHGCDTIGELYH-NEHAPNHD Integrin-binding domain

$$
+\quad+
$$

$+$

APEDLER-HAGDFGNVADYQGVATIYIDDSQVSLDPSSEAYIGGLAIVVHAGVDDLGRG-GNP---GPNDRIK-HIGDLGNIVAGANGVAEVYINSYDIKLR-GPLSVIG-HSLVVHANTDDLGQGTGNMREEAPDDSNR-HIGDLGNIESPASGDTLISVSDSLASLS-GQYSIIG-RSVVIHEKTDDLGRGTSDQ-----_----QHPGDFGNFAVRD-GSLWRYRAGLAASLA-GPHSIVG-RAVVHAGEDDLGRG-GNQ----_----QHPGDFGNFVVRD-GRLWKHRMGLATSLA-GPHSILG-RAVVVHAGEDDLGKG-GNQ---NPGDLGDLHDDDHGEVNATRTFD-WLTIGHTDGILGLSSAILQG------HTDGI-LGLSSAILQGDH

ESAKTGNAGARSGCGIIRVAPTYQPPQSGYRPRRPQHPNRQPGFPQQFQYQRTYN

-SLKTGNAGSRLACGVIGIAAVS

-SKTTGNAGSRLACGTIGTVEERILETTTASLPPVTQSQPIGSSSYYYSTFYLPIILYFLLSRIL ASVENGNAGRRLACCVVGVCGPGLWERQAREHSERKKRRRESECKAASVQNGNAGRRLACCVVGTSNSEAWESQTKE---RKKRRRESECKTTTSHT---A--VIACCVIGRSHAH-- 


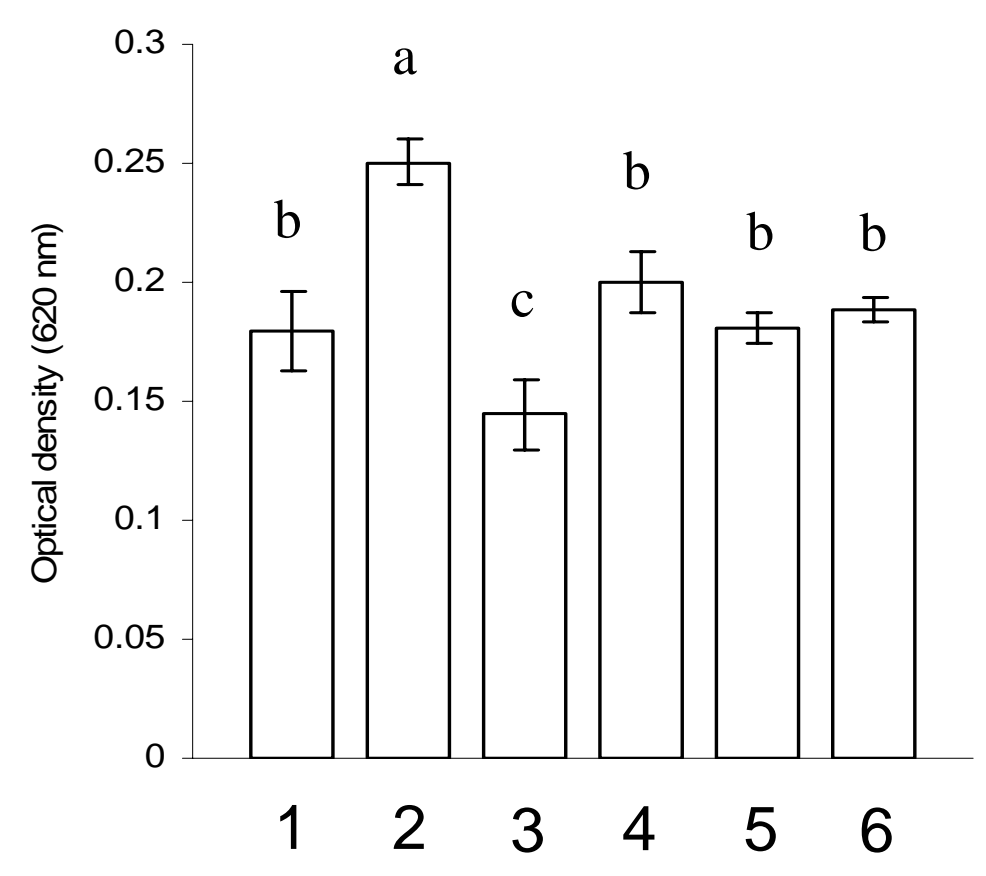

Figure 3 
Figure 4
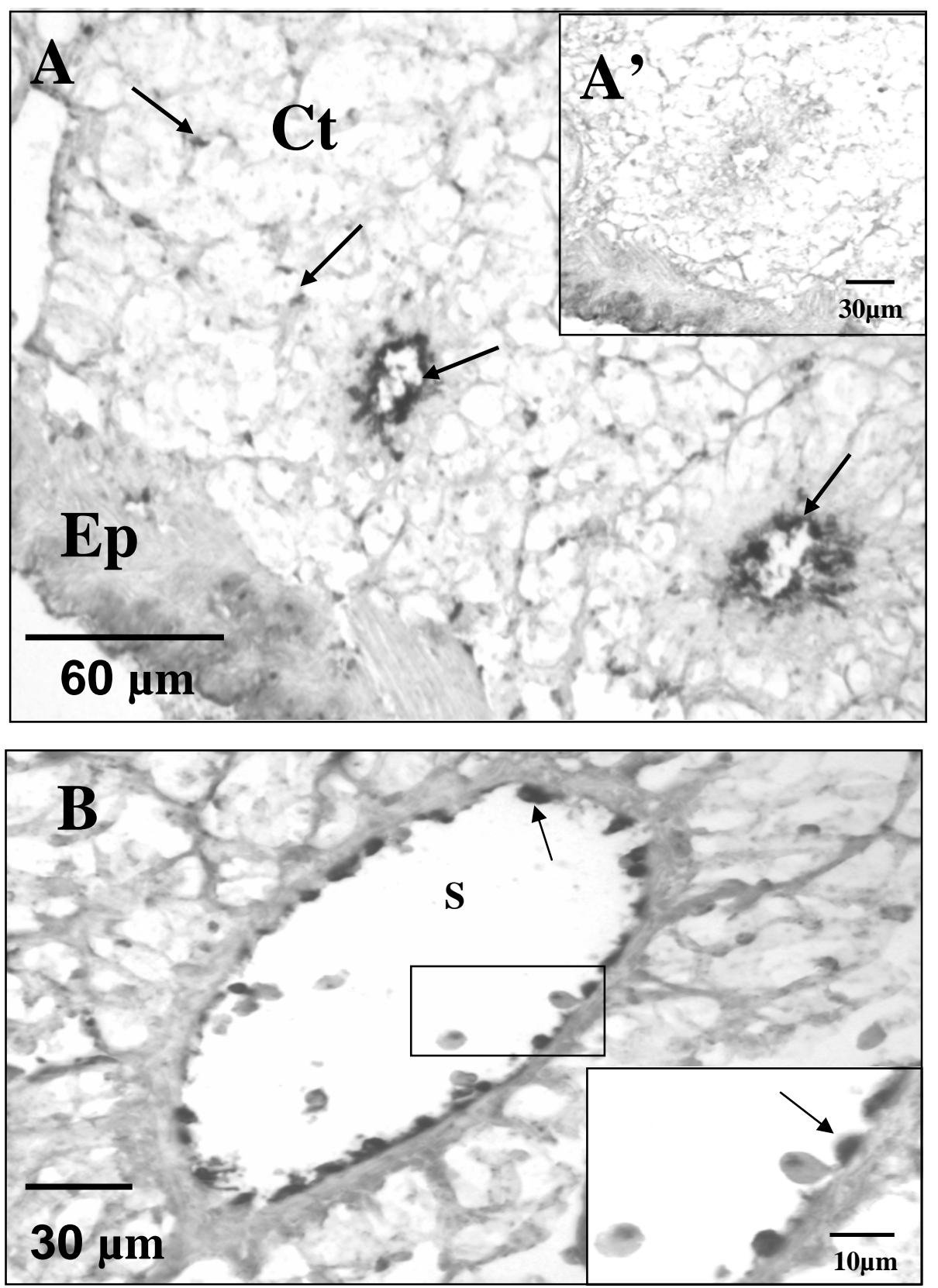
Figure 5
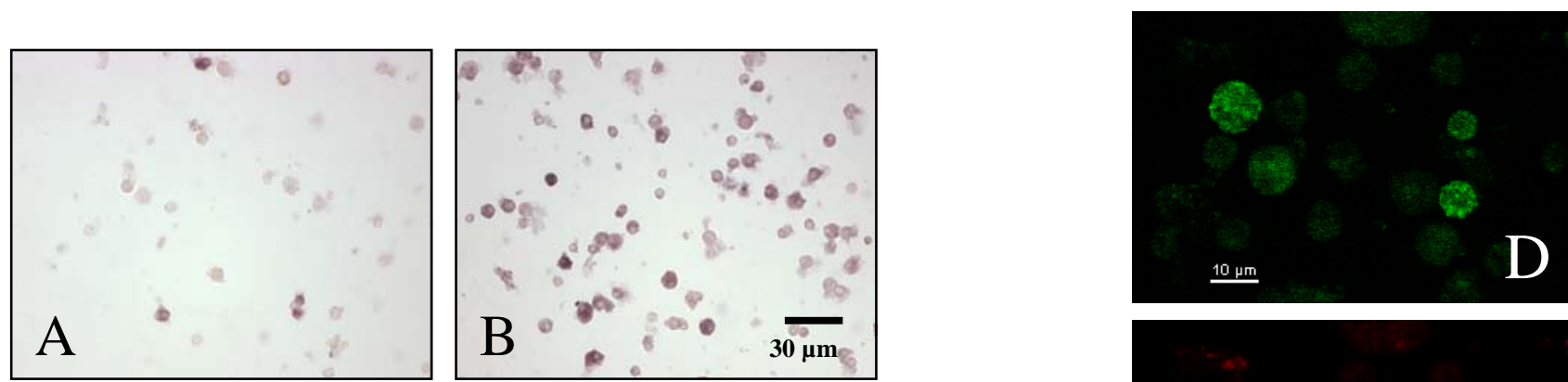

C

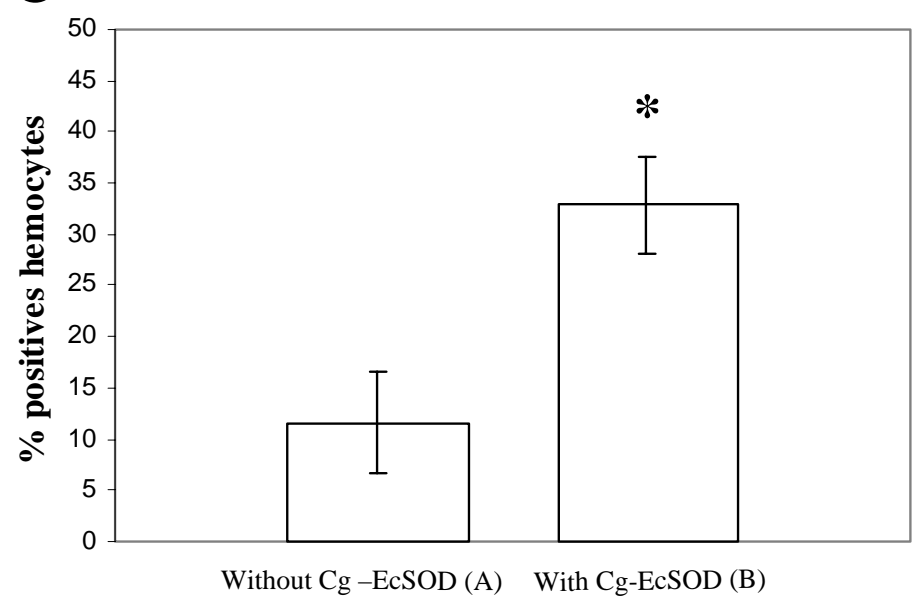

Treatments
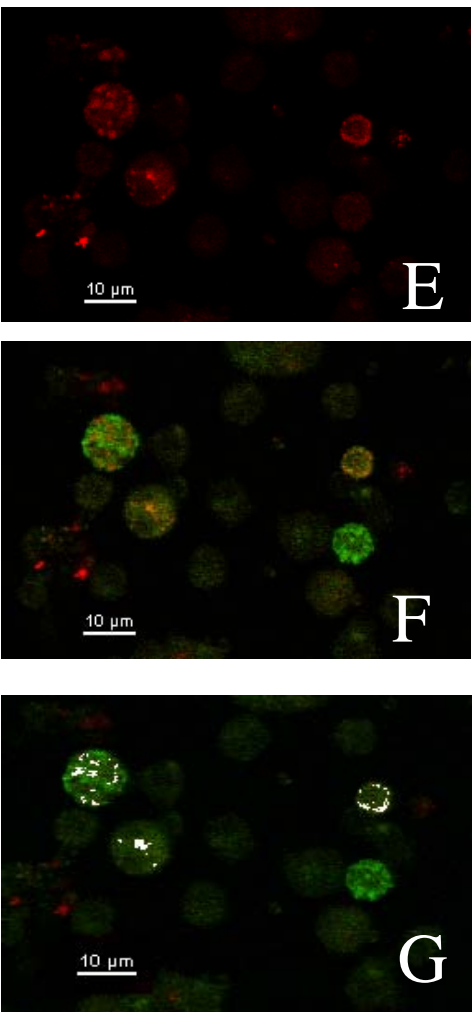


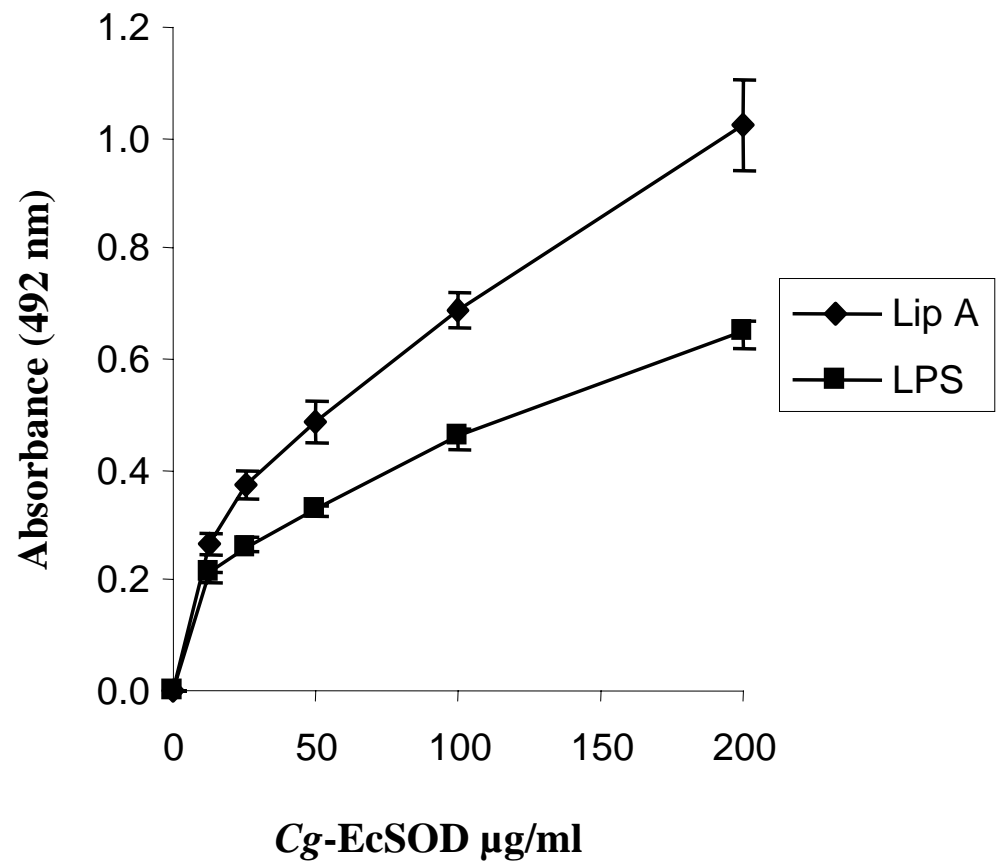

Figure 6 